\title{
Flexoelectricity and piezoelectricity - reason for rich variety of phases in antiferroelectric liquid crystals
}

\author{
${ }^{+\dagger}$ Mojca Čepič and ${ }^{+}$Boštjan Žekš \\ + J. Stefan Institute, Jamova 39, 1000 Ljubljana, Slovenia \\ ${ }^{\dagger}$ Faculty of Education, Kardeljeva pl. 16, 1000 Ljubljana, Slovenia \\ ${ }^{\diamond}$ Institute of Biophysics, Medical Faculty, Lipičeva 2, 1000 Ljubljana, Slovenia
}

(October 29, 2018)

\begin{abstract}
The free energy of antiferroelectric liquid crystal which takes into account polar order explicitly is presented. Steric, van der Waals, piezoelectric and flexoelectric interactions to the nearest layers and dipolar electrostatic interactions to the nearest and to the next nearest layers induce indirect tilt interactions with chiral and achiral properties, which extend to the third and to the fourth nearest layers. Chiral indirect interactions between tilts can be large and induce helicoidal modulations even in systems with negligible chiral van der Waals interactions. If indirect chiral interactions compete with chiral van der Waals interactions, the helix unwinding is possible. Although strength of microscopic interactions change monotonically with decreasing temperature, effective interlayer interactions change nonmonotonically and give rise to nonmonotouous change of modulation period through various phases. Increased enatiomeric excess i.e. increased chirality changes the phase sequence.
\end{abstract}

PACS numbers: $61.30 . \mathrm{Cz}$

Chiral polar smectics are materials which form layered phases. In these phases the average direction of elongated molecules is tilted for an angle with respect to the layer normal. Smectic layers are spontaneously polarized in the plane of the layer and perpendicular to the direction of the tilt [1]. Among these polar smectics the most widely experimentally and theoretically studied materials are ferroelectric liquid crystals. Ten years ago at an attempt to synthesise ferroelectric liquid crystals with larger polarization, antiferroelectric liquid crystals were discovered [2]. Their synthesis stimulated extensive experimental research which resulted in a discovery of many new polar liquid crystalline phases. In addition to the the ferroelectric Sm $\mathrm{C}^{*}$ phase and the antiferroelectric $\mathrm{Sm} \mathrm{C}_{A}^{*}$ phase, the $\mathrm{Sm} \mathrm{C}_{\alpha}^{*}$ phase and the $\mathrm{Sm} \mathrm{C}_{\gamma}^{*}$ phase were found to exist in some materials while in others instead of the $\mathrm{Sm} \mathrm{C}_{\gamma}^{*}$ phase, two phases - the Sm $\mathrm{C}_{F I 2}^{*}$ phase and the $\mathrm{Sm} \mathrm{C}_{F I 1}^{*}$ phase are present. The phases are modulated along layer normal and the modulation period of these phases was determined by resonant X-ray scattering [3]. The period was shown to vary nonmonotonically with decreasing temperature from a few layers in the $\mathrm{Sm} \mathrm{C}_{\alpha}^{*}$ phase, to a few hundred layers in the $\mathrm{Sm} \mathrm{C}^{*}$ phase, to approximately four layers in the $\mathrm{Sm} \mathrm{C}_{F I 2}^{*}$ phase, to approximately three layers in the Sm $\mathrm{C}_{F I 1}^{*}$ phase and to approximately two layers in the $\mathrm{Sm} \mathrm{C} \mathrm{C}_{A}^{*}$ phase at low temperatures.

Antiferroelectric liquid crystals can be theoretically described by continuous models [4 [- that predict only two of experimentally observed phases, the ferroelectric $\mathrm{Sm} \mathrm{C}^{*}$ phase and the antiferroelectric $\mathrm{Sm} \mathrm{C}_{A}^{*}$ phase. Discrete models [7 9] take interlayer interactions between the tilt vectors explicitly into account. The model [7] considers competing interactions between tilts in near- est layers and in next nearest layers and predicts various structures where the direction of the tilt varies uniformly from layer to layer. The model was later called the clock model [3].

Within this model the observed nonmonotonous temperature dependence of the modulation period can be reproduced only with nonmonotonous temperature dependences of model parameters, which cannot be understood from microscopic interactions. Although the model can predict structures of the $\mathrm{Sm} \mathrm{C}_{F I 2}^{*}$ and of the $\mathrm{Sm} \mathrm{C}_{F I 1}^{*}$ for the specific set of clock model parameters, these phases cannot exist as stable phases in a broader temperature interval. For this some synclinic interactions would be needed between the third and between the fourth nearest layers 97.

In this letter we present the model where the polarization of smectic layers is taken explicitly into account and is treated as a secondary order parameter to the tilt. In addition to the steric and van der Waals interactions to the nearest layers between the tilt vectors we consider also piezoelectric and flexoelectric couplings between the tilt and the polarization and electrostatic interactions between the polarizations of the nearest layers and polarizations with the next nearest layers [10].

To describe the structure of phases, we introduce the tilt vector $\boldsymbol{\xi}_{j}$ which gives the magnitude and the direction of the tilt in the $j$-th smectic layer and the polar order parameter $\boldsymbol{\eta}_{\boldsymbol{j}}=\left\{\eta_{j x}, \eta_{j y}\right\}$ which gives the magnitude and the direction of the in plane transverse polar order in the $j$-th layer. The spontaneous polarization in the $\mathrm{j}$ th layer is proportional to the polar order $\boldsymbol{\eta}: P_{j}=P_{0} \boldsymbol{\eta}_{j}$, where $P_{0}$ is a layer polarization for a completely polarly ordered layer 11. Therefore polar order parameter $\boldsymbol{\eta}$ will be here shortly called polarization. The free energy of the 
smectic system with $\mathrm{N}$ layers can be expressed in terms of both order parameters as:

$$
\begin{aligned}
\tilde{G} & =\sum_{j=1}^{N} \frac{1}{a} G_{0}+\frac{1}{2} a_{1}\left(\boldsymbol{\xi}_{j} \cdot \boldsymbol{\xi}_{j+1}\right)+\frac{1}{2} f_{1}\left(\boldsymbol{\xi}_{j} \times \boldsymbol{\xi}_{j+1}\right)_{z}+ \\
& +\frac{1}{2} b_{0} \boldsymbol{\eta}_{j}^{2}+\frac{1}{2} b_{1}\left(\boldsymbol{\eta}_{j} \cdot \boldsymbol{\eta}_{j+1}\right)+\frac{1}{8} b_{2}\left(\boldsymbol{\eta}_{j} \cdot \boldsymbol{\eta}_{j+2}\right)+ \\
& +c_{p}\left(\boldsymbol{\eta}_{j} \times \boldsymbol{\xi}_{j}\right)_{z}+\frac{1}{2} \mu\left(\boldsymbol{\xi}_{j+1}-\boldsymbol{\xi}_{j-1}\right) \cdot \boldsymbol{\eta}_{j} .
\end{aligned}
$$

Here $G_{0}=1 / 2 a\left(T-T_{0}\right) \xi_{j}^{2}+1 / 4 b \xi_{j}^{4}$ is the tilt dependent free energy of an isolated layer, where a continuous transition to the tilted phase takes place at $T_{0}$. Dividing all terms by the parameter $a$, we express all model parameters as well as the free energy $\tilde{G}$ in the units of temperature. Parameter $a_{1}$ corresponds to steric and van der Waals interactions between nearest layers. Steric interactions favor synclinic molecular alignment in neighboring layers and give a negative contribution to $a_{1}$, while van der Waals intermolecular interactions favor anticlinic ordering in neighboring layers and give a positive contribution to $a_{1}$. The parameter $a_{1}$ is of the order of a few Kelvins 88,11]. We believe that steric and van der Waals interactions are negligible further than to the nearest layers. The parameter $f_{1}$ gives the chiral part of van der Waals interactions between molecules 12] in neighboring layers and vanishes in racemic mixtures. We assume that $f_{1}$ is smaller than $a_{1}$ and it is a few tenths of a Kelvin. Polar vectors are associated with the layer polarization via magnitude and direction of molecular dipole moments. The parameter $b_{0}$ is positive because polarization is not a proper order parameter and both $b_{1}$ and $b_{2}$ favor antiparallel dipolar order and are therefore positive [10]. Since they give intralayer and interlayer interactions which decrease for more distant layers we expect that $b_{0}>>b_{1}>>b_{2}$. Transition temperatures from the $\mathrm{Sm} \mathrm{C}^{*}$ phase to the $\mathrm{Sm} \mathrm{C}_{A}^{*}$ phase are few degrees lower in nonpolar racemic mixtures than in pure samples [2] which suggests that $b_{0}$ is also of the order of a few $\mathrm{K}$. Polarization is induced in chiral polar smectics by the tilt and the piezoelectric coupling is given by $c_{p}$. The parameter $c_{p}$ has also a chiral character and vanishes in racemic mixtures. The magnitude of the layer polarization is only one tenth of the polarization of completely polarly ordered system [13] i.e. the magnitude of $\boldsymbol{\eta}_{j}$ is of the order 0.1 which means that also the value of $c_{p}$ is of the order of a Kelvin. The polarization coupling with the tilt in neighboring layers is given by $\mu$ and is of the same order as $c_{p}$ 14, 15.

The polar part of the free energy Eq. (11), can be written in the form

$$
G_{P}=\boldsymbol{\eta} \cdot \underline{C} \cdot \boldsymbol{\xi}+\frac{1}{2} \boldsymbol{\eta} \cdot \underline{B} \cdot \boldsymbol{\eta} .
$$

The tilt vector is $2 N$-dimensional vector of the form $\boldsymbol{\xi}=\left\{\xi_{1 x}, \ldots \xi_{j x}, \ldots \xi_{N x}, \xi_{1 y}, \ldots \xi_{N y}\right\}$ and similar for the polarization $\boldsymbol{\eta}$. The five-diagonal $2 N$-dimensional matrix $\underline{B}$ gives intralayer and interlayer electrostatic interactions with elements $B_{j, j}=b_{0}, B_{j, j \pm 1}=\frac{1}{2} b_{1}$ and $B_{j, j \pm 2}=\frac{1}{8} \quad b_{2}$. The $2 N$-dimensional matrix $\underline{C}$ gives the coupling between tilts and polarizations. The offdiagonal elements $C_{j, j \pm 1}=C_{j+N, j+N \pm 1}=\frac{1}{2} \mu$ and $C_{j, j+N}=-C_{j+N, j}=c_{p}$. Minimization of Eq. (2) with respect to polarization $\boldsymbol{\eta}$ gives

$$
\boldsymbol{\eta}=-\underline{B}^{-1} \cdot \underline{C} \boldsymbol{\xi}
$$

where $\underline{B}^{-1}$ is the inverse matrix of the five-diagonal matrix $\underline{B}$. As $b_{0}>>b_{1}>>b_{2}$ we keep in $\underline{B}^{-1}$ only the terms up to the second order in $b_{1} / b_{0}$ and up to the first order in $b_{2} / b_{0}$. The elements of $\underline{B}^{-1}$ are $B_{j, j}^{-1}=\frac{1}{b_{0}}\left(1+\frac{1}{2}\left(\frac{b_{1}}{b_{0}}\right)^{2}\right), B_{j, j \pm 1}^{-1}=-\frac{1}{2 b_{0}}\left(\frac{b_{1}}{b_{0}}\right)$ and $B_{j, j \pm 2}^{-1}=\frac{1}{8 b_{0}}\left(2\left(\frac{b_{1}}{b_{0}}\right)^{2}-\left(\frac{b_{2}}{b_{0}}\right)\right)$. Inserting the tilt dependent polarization Eq. (3) into Eq. (2) we obtain the polar part of free energy as

$$
G_{P}=-\frac{1}{2} \boldsymbol{\xi} \underline{C} \underline{B}^{-1} \underline{C} \boldsymbol{\xi}
$$

and the free energy due to interlayer interactions is

$$
\tilde{G}_{\text {int }}=\frac{1}{2} \sum_{j=1}^{N}\left(\sum_{k=1}^{4} \tilde{a}_{k}\left(\xi_{j} \cdot \xi_{j+k}\right)+\sum_{k=1}^{3} \tilde{f}_{k}\left(\xi_{j} \times \xi_{j+k}\right)\right) .
$$

Parameters $\tilde{a}_{k}$ and $\tilde{f}_{k}$ which appear after polarization elimination, give effective interactions between tilts. Although direct van der Waals and steric interactions are significant only to the nearest layers and electrostatic interactions are significant up to the next nearest layers, effective interactions are significant up to the fourth nearest layers.

$$
\begin{aligned}
& \tilde{a}_{1}=a_{1}+\left(\frac{c_{p}^{2}}{b_{0}}+\frac{1}{4} \frac{\mu^{2}}{b_{0}}\right)\left(\frac{b_{1}}{b_{0}}\right) \\
& \tilde{a}_{2}=\frac{1}{2} \frac{c_{p}^{2}}{b_{0}}\left(\frac{1}{2}\left(\frac{b_{2}}{b_{0}}\right)-\left(\frac{b_{1}}{b_{0}}\right)^{2}\right)+\frac{1}{2} \frac{\mu^{2}}{b_{0}}\left(1+\frac{1}{4}\left(\frac{b_{2}}{b_{0}}\right)\right) \\
& \tilde{a}_{3}=-\frac{1}{4} \frac{\mu^{2}}{b_{0}}\left(\frac{b_{1}}{b_{0}}\right) \\
& \tilde{a}_{4}=\frac{1}{8} \frac{\mu^{2}}{b_{0}}\left(\left(\frac{b_{1}}{b_{0}}\right)^{2}-\frac{1}{2}\left(\frac{b_{2}}{b_{0}}\right)\right) \\
& \tilde{f}_{1}=f_{1}-\frac{2 c_{p} \mu}{b_{0}}\left(1+\frac{1}{4}\left(\frac{b_{1}}{b_{0}}\right)^{2}+\frac{1}{8}\left(\frac{b_{2}}{b_{0}}\right)\right) \\
& \tilde{f}_{2}=\frac{c_{p} \mu}{b_{0}}\left(\frac{b_{1}}{b_{0}}\right) \\
& \tilde{f}_{3}=\frac{1}{2} \frac{c_{p} \mu}{b_{0}}\left(-\left(\frac{b_{1}}{b_{0}}\right)^{2}+\frac{1}{2}\left(\frac{b_{2}}{b_{0}}\right)\right) .
\end{aligned}
$$


Achiral effective interactions between nearest layers $\tilde{a}_{1}$ consist of direct steric and van der Waals interactions $\left(a_{1}\right)$ as well as of indirect interactions due to piezoelectrically and flexoelectrically induced polarization. Achiral interaction between next nearest layers are only indirect and can be for systems with negligible flexoelectric interaction $(\mu \approx 0)$ either competing [7] $\left(\tilde{a}_{2}>0\right)$ or noncompeting with $\left(\tilde{a}_{2}<0\right)[16$. The second case leads to the validity of the continuous bilayer models [ [ 6 ], although it seems that the present experimental knowledge supports this possibility by a single experimental evidence [16]. In systems with large flexoelectric interactions $\left(\left|c_{p}\right| \frac{b_{1}}{b_{0}}<|\mu|\right)$, $\tilde{a}_{2}$ is always positive and competes with interactions between nearest layers $\tilde{a}_{1}$. Indirect effective interactions between third neighbors are given by $\tilde{a}_{3}$. The term is always negative and favors synclinic tilt directions in interacting layers. Therefore this term in systems with significant flexoelectric interactions tends to stabilize the structures with three layer periodicity. Indirect effective interactions between fourth nearest layers $\tilde{a}_{4}$ can be either negative or positive.

The effective chiral coupling between neighboring layers has two contributions: the van der Waals originated $\left(f_{1}\right)$ and the polarization part which appears due to the combination of piezo and flexoelectricity $\left(c_{p} \mu / b_{0}\right)$. Even systems with negligible direct chiral interactions $f_{1} \approx 0$ are hellicoidally modulated. In all previous models, chiral interactions have always been treated as weak interactions which induce only slight perturbations in the structure i.e. helicoidal modulations with very long periods. In antiferroelectric liquid crystals this is not always the case. The ratio $c_{p} \mu / b_{0}$ can have the value of a few Kelvins and is comparable to other achiral interlayer interactions. The competition between the van der Waals part of chiral interactions $\left(f_{1}\right)$ and indirect chiral interactions due to piezoelectric and flexoelectric effect $\left(c_{p} \mu / b_{0}\right)$ can explain the helix unwinding without polarization reversal as observed in the antiferroelectric phase of various systems [2, 16].

The simplest solution, which minimizes the free energy Eq. (我) for competing interlayer interactions $\left(\tilde{a}_{2}>0\right)$, is the clock model solution with the constant phase difference between neighboring layers over the whole sample [7]. The tilt in the $\mathrm{j}$-th layer is $\xi_{j}=\theta\{\cos (j \alpha), \sin (j \alpha)\}$ where $\theta$ is constant and the phase difference $\alpha$ is the difference in directions of the tilt vectors in neighboring layers. The phase difference $\alpha$ is obtained by the minimization of the free energy Eq. (4) with respect to $\alpha$.

We have analyzed the temperature dependence of solutions for the following behavior of model parameters with decreasing temperature: $a_{1}$ increases monotonically from the negative value to the positive value, piezoelectric parameter $c_{p}$ monotonically increases and flexoelectric parameter $\mu$ monotonically decreases. Let us consider a microscopic mechanism of the described parameter dependence with decreasing temperature. The monotonous increase of direct interactions $a_{1}$ with decreasing temperature from negative value which is due to interpenetrating molecules through nearest layers, to the positive value in the region where van der Waals interactions favor anticlinic order, is due to increasing smectic order [18]. The flexoelectric coupling $(\mu)$ decreases monotonically with decreasing temperature, since the smectic order and hindrance of the rotation becomes less affected by the molecules above and below the interacting layer. In contrast, with decreasing temperature piezoelectric coupling $\left(c_{p}\right)$ monotonically increases since higher smectic order strengthen the rotation hindrance within the layer.

For positive $\tilde{a}_{2}$ we can introduce the measure of the competition - the competition ratio $4 \tilde{a}_{1} / \tilde{a}_{2}$. In racemic mixtures in the temperature region where the competition ratio is smaller than -1 , the synclinic nonmodulated $\mathrm{Sm} \mathrm{C} \mathrm{C}^{*}$ phase is stable. In the temperature region where the competition ratio is larger than 1 , the anticlinic nonmodulated $\mathrm{Sm} \mathrm{C}_{A}^{*}$ phase exists. In the region in between, the phase angle $\alpha$ changes with decreasing temperature rapidly from $0^{\circ}$ to $180^{\circ}$ i.e. modulation period changes from infinity to two layers. In slightly chiral systems the $\mathrm{Sm} \mathrm{C}_{\alpha}^{*}$ phase appears above the ferroelectric Sm $\mathrm{C}^{*}$ phase. With additional increase of the enantiomeric excess or increased chirality of the system (larger $c_{p}$ ), the ferroelectric Sm C* phase dissapear, leaving only modulated phase with short pitch (Fig. 1).

Experimental observations have shown that in racemic mixtures only the synclinic Sm $\mathrm{C}^{*}$ phase and the anticlinic Sm $\mathrm{C}_{A}^{*}$ phase exist with the first order transition between them [2]. The narrow temperature range of the modulated phase (Fig. 1 - solid line), can be experimentally seen as the first order transition. In chiral samples two additional phases the $\mathrm{Sm} \mathrm{C}_{\alpha}^{*}$ phase and the $\mathrm{Sm} \mathrm{C}_{\gamma}^{*}$ phase appear. In chiral systems where piezoelectric coupling $c_{p}$ is important, below the transitions temperature to the tilted phase, the modulation period is much shorter and increases with decreasing temperature. The temperature region where competition ratio is between -1 and +1 becomes wider and can be experimentally observed as a distinguished $\mathrm{Sm} \mathrm{C}_{\gamma}^{*}$ phase. The similar behavior is observed in chiral samples of $\mathrm{MH}$ POBC [2] and 10OTBBB1M7 [3] (Fig. 1 - dashed line). If piezoelectric $c_{p}$ coupling is still stronger, the ferroelectric $\mathrm{Sm} \mathrm{C}^{*}$ phase dissapears, which was observed in some systems 17] (Fig. 1 - dotted line). The present form of the model takes into account only quadratic interactions and can not account for first order transitions between phases with different values of $\alpha$.

The symmetry of structures with various $\alpha$ is the same, since the symmetry operation common to all structures consists from the translation for a layer thickness and the rotation for an angle $\alpha$. If $\alpha$ changes discontinuously, the temperature range of different phases can be defined with DSC or similar measurements. If transitions are continuous, the temperature ranges of various phases are 
defined by changes of the macroscopic properties and are to some extent arbitrary. The phase difference $\alpha$ can be used as a parameter which distinguishes between different phases. In the $\mathrm{Sm} \mathrm{C}_{\alpha}^{*}$ phase varies from $\alpha \approx 10^{\circ}$ to $70^{\circ}$ which means that the modulation period varies from 40 to 5 layers. In the $\mathrm{Sm} \mathrm{C}^{*}$ phase is $\alpha \approx 0$ i.e. the infinite modulation period in racemic mixtures and a few hundred layers in pure samples. In the $\mathrm{Sm} \mathrm{C}_{F I 2}^{*}$ phase is $\alpha \approx 90^{\circ}$ or four layers, in the $\mathrm{Sm} \mathrm{C}_{F I 1}^{*}$ phase is $\alpha \approx 120^{\circ}$ or three layers. In the $\mathrm{Sm} \mathrm{C}_{A}^{*}$ phase is $\alpha \approx 180^{\circ}$. The modulation period is two layers and can also be analyzed within a bilayer model [4 [6] where it is treated as a long double helix.

To conclude, we present the phenomenological model based on microscopic intralayer and interlayer interactions. Polarization is induced by piezoelectric and flexoelectric effect and is taken into account explicitly. Although tilts directly interact only with nearest layers and polarizations interact up to the next nearest layers, indirect achiral interactions which tend to stabilize structures with three layer and four layer periodicities extend to the fourth neighboring layers. Chiral piezoelectric couplings influence also the effective achiral interactions between nearest layers. Additionally, chiral interactions due to the flexoelectric effect are important to the third nearest layers and can be strong. Direct interactions between nearest layers correspond to elastic terms in continuous models. As a consequence of the piezoelectric and flexoelectric coupling, the corresponding Lifshitz parameter in continuous models is renormalized. In contrast, variation of polarization, which is considered as electrostatic interlayer coupling, has no corresponding terms in continuous models. The large variety of phases in antiferroelectric liquid crystals is a consequence of a delicate balance between various mechanisms. In chiral samples all interactions change with temperature monotonically. But the competition ratio varies nonmonotonically and therefore also phase difference $\alpha$ i.e. modulation period changes nonmonotonically and the ferroelectric $\mathrm{Sm} \mathrm{C*}$ phase with long modulation period can be stable between two phases $\left(\mathrm{Sm} \mathrm{C} \mathrm{C}_{\alpha}^{*}\right.$ and $\left.\mathrm{Sm} \mathrm{C}_{F I 2}^{*}\right)$ with much shorter modulation periods.

Within the phenomenological discrete model we studied only the simplest possible interlayer interactions and the simplest possible clock model solutions. As a future problem we left the analysis of the influence of the phase difference $\alpha$ on the tilt which could induce first order transitions between phases. We also did not consider quadrupolar coupling, which could induce quadrupolar ordering and therefore allow for distorted clock model solutions 19].

Authors are very grateful to Ewa Gorecka and Nataša Vaupotič for many stimulating discussions. The financial support of Ministry of Education, Science and Sport of Slovenia, grant PO-518 is acknowledged.
[1] D.J. Photinos, E.T. Samulski, Science 270, 783 (1995).

[2] A.D.L.Chandani, E. Gorecka, Y. Ouchi, H. Takezoe, A. Fukuda, Jpn. J. Appl. Phys., 28, 1265 (1989).

[3] P. Mach, R. Pindak, A.-M. Levelut, P. Barois, H.T. Nguyen, C.C. Huang, and L. Furenlid, Phys. Rev. Lett. 81, 1015 (1998).

[4] H. Orihara, Y. Ishibashi, Jpn. J. Appl. Phys. 30, L1819 (1990).

[5] B. Žekš, M. Čepič, Liq. Cryst. 14, 445 (1993).

[6] V.L.Lorman, A.A.Bulbitch, P.Toledano, Phys. Rev.E49. 1367 (1994).

[7] M. Čepič, B. Žekš, Mol. Cryst. Liq. Cryst. 263, 61 (1995).

[8] M. Škarabot, M. Čepič, B. Žekš, R. Blinc, G. Heppke, A.V. Kytik, I. Muševič, Phys. Rev. E 58, 575 (1998).

[9] A. Roy, N.V.Madhusudana, Eur.Phys.J.E 1, 319 (2000).

[10] M. Čepič, B. Žekš, Mol. Cryst. Liq. Cryst. 301, 221 (1997).

[11] D.R. Link, private communication.

[12] A.B. Harris, Randall D. Kamien, T.C. Lubensky, Phys. Rev. Lett. 78, 1476 (1997).

[13] I. Muševič, R. Blinc, B. Žekš, The physics of ferroelectric and antiferroelectric liquid crystals, World Scientific, Singapore (2000).

[14] P.O. Andreeva, V.K. Dolganov, C. Gors, R. Fouret, E.I. Kats, Phys. Rev. E 59, 4143 (1999).

[15] D.R. Link, G. Natale, J.E. Maclennan, N.A. Clark, M. Walsh, S.S. Keast, M.E. Neubert, Phys. Rev. Lett. 83, 3665 (1999).

[16] I. Muševič, A. Rastegar, M. Čepič, B. Žekš, M. Čopič, D. Moro, G. Heppke, Phys. Rev. Lett. 77, 1796 (1996).

[17] M. Čepič, G. Heppke, J.M. Hollidt, D. Loetsch, D. Moro, B. Žekš, Mol. Cryst. Liq. Cryst. 263, 207 (1995).

[18] D. Pociecha, E. Gorecka, M. Čepič, N. Vaupotič, B. Žekš, D. Kardas and J. Mieczkowski, Phys. Rev. Lett. 86, 3048 (2001).

[19] P.M. Johnson, D.A. Olson, S. Pankratz, T. Nguyen, J. Goodby, M. Hird, C.C. Huang, Phys. Rev. Lett. 84, 4870 (2000).

FIG. 1. Temperature dependence of the phase difference $\alpha$. Model parameters change with temperature monotonically as: $a_{1}=(-4.1 \mathrm{~K}-3.2 \mathrm{~T}), c_{p}=x(0.15 \mathrm{~K}-0.16 \mathrm{~T})$, $\mu=(2.12 \mathrm{~K}+1.32 \mathrm{~T})$. The other values were $f=0 \mathrm{~K}$; $b_{0}=2 \mathrm{~K} ; b_{1}=b_{0} / 10 ; b_{2}=b_{0} / 100$. The parameter $x$ gives enantiomeric excess and has the following values: $x=0$ solid line, $x=0.2$ - dashed line and $x=1$ - dotted line. 
This figure "fig1.png" is available in "png" format from: http://arxiv.org/ps/cond-mat/0104508v1 